AFCRL-

Calculations of Reflected and Transmitted Radiance for Earth's Atmosphere

Authors

Gilbert N. Plass

George W. Kattawar

Southwest Center for Advanced Studies Dallas, Texas

Contract No. AFl9(628)-5039

Project No. 4076

Task No. $\quad 407604$

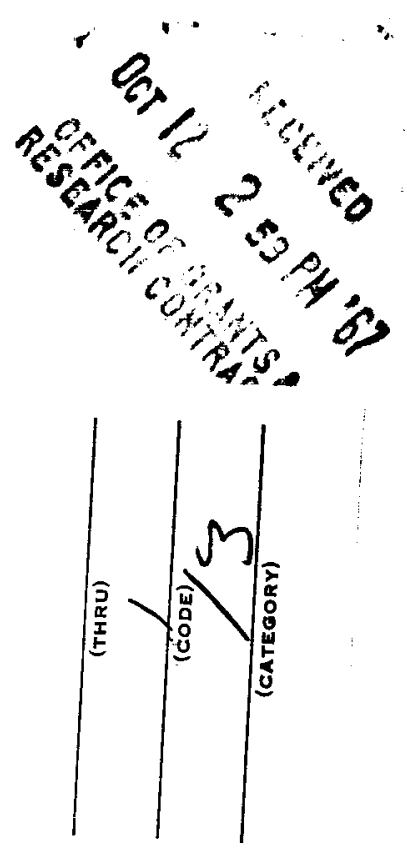

Dallas, Texas
Contract No. AFl9(628)-5039
Project No. 4076
Task No.
407604

Scientific Report No. 6

13 September 1967

This research was partially supported by the National Aeronautics and Space Administration

Contract Monitor

Robert W. Fenn

Optical Physics Laboratory

Distribution of this document is unlimited. It may be released to the Clearinghouse, Department of Commerce, for sale to the general public.

$$
\begin{gathered}
\text { Prepared } \\
\text { for }
\end{gathered}
$$

AIR FORCE CAMBRIDGE RESEARCH IABORATORIES

OFFICE OF AEROSPACE RESEARCH

UNITED STATES AIR FORCE

BEDFORD, MASSACHUSETTS 01730 
AFCRL-

\author{
Calculations of Reflected and Transmitted \\ Radiance for' Earth's Atmosphere
}

Authors

Gilbert N. Plass

George W. Kattawar

Southwest Center for Advanced Studies
Dallas, Texas

Contract No. AF19(628)-5039

Project No. 4076

Task No. $\quad 407604$

Scientific Report No. 6

13 September 1967

This research was partially supported by the National Aeronautics and Space Administration

Contract Monitor

Robert W. Fenn

Optical Physics Laboratory

Distribution of this document is unlimited. It may be released to the Clearinghouse, Department of Commerce, for sale to the general public.

Prepared

for

AIR FORCE CAMBRIDGE RESEARCH LABORATORIES

OFFICE OF AEROSPACE RESEARCH

UNITED STATES AIR FORCE

BEDFORD, MASSACHUSETTS 01730 


\title{
CALCULATIONS OF REFLECTED AND TRANSMITTED \\ RADIANCE FOR EARTH'S ATMOSPHERE
}

\author{
by \\ Gilbert N. Plass and George W. Kattawar \\ Southwest Center for Advanced Studies \\ Dallas, Texas 75230
}

September 13, 1967

To be submitted to

Applied Optics

This work was supported by the Air Force Cambridge Research Laboratories, Office of Aerospace Research Contract AF19(628) - 5039 and by the National Aeronautics and Space Administration contract. 
Introduction

The radiance of the earth's atmosphere is determined by the multiple scattering of photons from the aerosols and molecules in the atmosphere and by the reflection properties of the ground. Calculations have previnisly been made for an atmosphere with only Rayleigh scattering and the ground represented by a Lambert's surface; the results have been published in an extremely useful set of tables ${ }^{1}$. Others have attempted to include the effect of aerosols whose scattering function has a strong forward maximum. Up to the present these calculations have either used an approximate scattering function (Feigelson et $\mathrm{al}^{2}$ ) or an approximation to the contributions from multiple scattering $\left(\right.$ Fraser $\left.^{3}\right)$.

With the Monte Carlo method for following the path of a multiple scattered photon, it is possible both to use the exact aerosol scattering function calculated from Mie theory and to obtain the contribution through any desired order of scattering. The statistical fluctuations in the result can be held to acceptable levels by following a sufficient number of photon histories. The Monte Carlo method which we use here has been described in detail in the literature ${ }^{4}$. A somewhat different Monte Carlo method has been applied to the real atmosphere at a wavelength of $0.55 \mu$ by collins et $a 1^{5}$. The calculations reported here do not include polarization effects. However, preliminary calculations with a Monte Carlo method using the Stokes vectors indicates that the intensities are nearly the same as in the linear theory when Mie scattering is important. 
Method

At each wavelength the Rayleigh attenuation coefficient and ozone absorption coefficient as a function of height are taken from the tables compiled by Elterman ${ }^{6}$ (p.7-14 to 7-35). Two different aerosol number density distributions with height are used: (I) Elterman's distribution as given on p. 7-2 of reference 6; (2) Kondratiev et al's distribution as given in Fig. 16 of reference 7 . The main differences between these distributions is that Elterman's has more aerosols in the lowest $2 \mathrm{~km}$ of the atmosphere and Kondratiev's has more aerosols at all altitudes above $6 \mathrm{~km}$. At all altitudes above $9 \mathrm{~km}$ Kondratiev's distribution has at least 10 times as many aerosols as Elterman's and at some altitudes has 20 times as many.

The aerosols are represented by the Haze $C$ model proposed by Deirmendjian ${ }^{8}$. The number density in this model is constant for $0.03<r<0.1 \mu$ and is proportional to $r^{-4}$ for $r>0.1 \mu$, where $r$ is the aerosol radius. The scattering function for this distribution of spherical particles was calculated exactly from the Mie theory ${ }^{9}$ for the wavelengths used in this calculation. The scattering function is shown in Fig. 1. The inset in the upper left portion of the figure shows the scattering function near $0^{\circ}$. The scattering angle for all aerosol collisions is chosen from this distribution. From these results the aerosol attenuation length as a function of altitude is calculated for both the Elterman and Kondratiev aerosol distributions. 
The total optical thickness of the atmosphere is calculated from the Rayleigh and aerosol attenuation coefficients and the ozone absorption coefficient. The atmosphere is divided into a number of layers and the ratio of the Rayleigh extinction to total cross sections and the scattering to extinction cross section for both the Mie and Rayleigh particles is established in each layer. The probability of ozone absorption is also calculated from the ozone absorption coefficient for each layer. All calculations are done with the optical depth as the parameter. Input data given as a function of altitude is changed to optical depth as the parameter for use in the program.

Radiance at $0.7 \mu$

The reflected and transmitted radiance at the top and bottom respectively of the atmosphere at $0.7 \mu$ is shown in Fig. 2 as a function of the cosine of the zenith angle $(\mu)$. The cosine of the solar zenith angle $\left(\mu_{0}\right)$ is -1 (sun at the zenith) for this figure. Curves are shown for various surface albedos A. A Lambert surface is assumed to represent the ground.

The radiance calculated for the Kondratiev et al ${ }^{7}$ and Elterman ${ }^{6}$ aerosol distributions are compared for $A=0$ and 1 . The differences between these two distributions are quite small and may largely be statistical fluctuations in the results. The differences are also small for intermediate values of the albedo. The optical depth $\tau$ of the atmosphere was chosen to be 0.1433 for both distributions in order that the effect of aerosol variations with height on the radiances 
could be studied for the same value of $\tau$. This is the $\tau$ value computed for the Kondratiev et $\mathrm{al}^{7}$ distribution without ozone absorption. The corresponding value for the Elterman distribution is $\tau=0.1836$ $(\tau=0.217$ with ozone absorption). The Elterman distribution was arbitrarily terminated at $\tau=0.1433$ near the ground for this comparison. Separate runs with and without the ozone absorption showed that it was sufficiently small to have no appreciable effect on the radiance at this wavelength.

In order to further compare the two aerosol distributions, the upward and downward radiance was computed at several different heights in the atmosphere corresponding to $\tau=0,0.005,0.01,0.02,0.05$, and 0.1433 ( $\tau$ a 0 corresponds to the top of the atmosphere and 0.1433 to the bottom). The results are shown in Fig. 3. The upward radiance is for $\mathrm{A}=0$ and the downward radiance is for $\mathrm{A}=0.4$ as perhaps these values best illustrate the differences in these aerosol distributions.

The upward radiances show the greatest differences between the two distributions at $\tau=0.05$ and 0.02 with the Kondratiev distribution always showing the larger radiance values. This is because more photons reach the lower levels of the atmosphere through numerous small angle forward scattering events from the aerosols with the Kondratiev distribution because of its greater aerosol number at the higher altitudes. Some of these photons are then scattered upward to contribute to the upward radiance.

The downward radiances for A 0.4 are always considerably larger for the Kondratiev distribution than for the Elterman distribution near $\mu \square I$ (except at the lower surface). This shows clearly the contribution from strong forward scattering of the additional aerosols for the 
Kondratiev distribution. At other angles the downward radiance is in general less for the Kondratiev distribution at the higher altitudes. It should be remembered that the same $\tau$ values correspond in general to different altitudes for different aerosol distributions. Thus, although different aerosol distributions can influence the radiance at intermediate heights in the atmosphere, they seem to give nearly the same results at the top and bottom of the atmosphere, provided only that the total optical thickness is the same.

The curves for the reflected and transmitted radiance for the entire atmosphere that are given in Fig. 2 are a combination of the typical curves for the Rayleigh and the Haze $C$ scattering function. 10 In particular the transmitted radiance for a Rayleigh scattering function never rises at the zenith; it is seen from Fig. 2 that the actual radiance increases at the zenith because of the aerosol scattering.

The reflected and transmitted radiance for $\mu_{0}=-0.1$ is given in Fig. 4. The marked asymmetrical shape of the reflected radiance curves about $\mu=1$ indicates the important contribution from aerosol scattering and can not be explained by pure Rayleigh scattering. An important parameter is the ratio of the maximum value (at $\mu=0$ on solar horizon) to the minimum value (near the zenith toward antisolar horizon) of the reflected radiance. This ratio is approximately 20 and 500 for Rayleigh and Haze $C$ scattering functions respectively. 10 The ratio for the earth's atmosphere as shown in Fig. 4 is 36 which shows the contribution from both scattering functions. 
Radiance at $0.4 \mu$

At a wavelength of $0.4 \mu$ the Rayleigh scattering is more important than the aerosol scattering at all altitudes above $2 \mathrm{~km}$. The calculated radiances are shown in Fig. 5 for $\mu_{0}=-1$. The optical depth of the atmosphere is 0.577 . The Elterman aerosol distribution is used in this and all the following calculations reported here. The reflected radiance is now more nearly the curve for pure Rayleigh scattering, although still modified by the aerosol scattering. The variation from zenith to horizon for $A=0$ is much less at $0.4 \mu$ than at $0.7 \mu$. The transmitted radiance varies near the horizon as would be expected for the Rayleigh scattering function, but also shows the characteristic increase due to aerosol scattering near the incident direction.

The reflected and transmitted radiance for $\lambda=0.4 \mu$ is shown in Fig. 6. The reflected radiance for $A=1$ increases from the nadir to the antisolar horizon. ${ }^{10}$ Thus the aerosol scattering has only a minor role in determining the radiance. The transmitted radiance of the atmosphere is nearly constant from one horizon to the other (characteristic of Rayleigh scattering) with a slight perturbation which produces a weak maximum around $\mu=0.3$ on the solar horizon and around $\mu=0.2$ on the antisolar horizon and a weak minimum near the zenith. This variation is characteristic of the transmitted radiance for the Haze $C$ model ${ }^{10}$ and is the contribution from the relatively small number of aerosols.

Radiance at $0.3 \mu$

At $0.3 \mu$ the ozone absorption is significant. The ozone absorption coefficient is larger than either the Rayleigh or aerosol attenuation 
coefficient at all altitudes from 10 to $50 \mathrm{~km}$. The values for the ozone absorption coefficient were taken from Elterman's tables ${ }^{6}$. The reflected radiances for $\mu_{0}=-1$ are shown in Fig. 7. The curves are the same for all surface albedos, since very little radiation can penetrate to the surface and back to space with the strong ozone absorption. The curve is very similar to the reflected radiance curvell for large $\tau$ and a single scattering albedo $\omega_{0} \sim 0.4$.

The transmitted radiance is shown in Fig. 8. There is a variation with surface albedo as an appreciable fraction of the radiation that reaches the surface is scattered by the lower atmospheric layers into the downward direction once again. These curves are again typical of scattering for large $\tau$ and a single scattering albedo $\omega_{0} \sim 0.4$. There are fairly large fluctuations in our results for both the reflected and transmitted radiance because of the small numerical values (indicating a small number of photons). However, the general trend of the curves is well established and the small numerical values are in themselves important.

The reflected radiance for $\mu_{0}=-0.1$ is shown in Fig. 9. The same general remarks apply here as for the case of $\mu_{0}=-1$. The transmitted radiance is too small to calculate.

Radiance at $0.27 \mu$

At $0.27 \mu$ the ozone absorption coefficient is larger than either the Rayleigh or aerosol attenuation coefficient at all altitudes. In turn the Rayleigh attenuation coefficient is langer than the aerosol coefficient 
at all altitudes above $1 \mathrm{~km}$. The optical thickness of the atmosphere is 73.25 . The reflected radiance for $\mu_{0}=-1$ and -0.1 is shown in Figs. 7 and 9 .

When there is Rayleigh scattering and strong absorption, the reflected radiance is 10 times as large at the horizon as at the nadir when $\mu_{0}=-0.1$ (Fig. 9), but less than twice as large when $\mu_{0}=-1$ (Fig. 7). The reason is that the first collision of the photon occurs on the average at the same optical depth as measured along the incident path for any angle of incidence. Thus the first collision is on the average 10 times nearer the surface when $\mu_{0}=-0.1$ than when $\mu_{0}=-1$. The scattered particle emitted according to the Rayleigh scattering function is on the average near an optical depth of 0.1 when $\tau=0.1$; when it is emitted upward it can escape from the atmosphere at most angles without undergoing further scattering events. Thus, the angular distribution is proportional to the Rayleigh scattering function divided by $\mu$ (geometrical factor). This is modified near the horizon by multiple scattering, but there is still a strong variation of the reflected radiance with $\mu$. On the other hand when $\mu_{0}=-1$, the first collision is on the average nean $\tau=1$. Only photons scattered backward toward the zenith direction can escape from the atmosphere with an appreciable probability without further scattering events. Photons scattered into other directions have a high probability of undergoing further scattering events. The resultant multiple scattering greatly reduces the variation of the reflected radiance with $\mu$. 
Radiance at $1.67 \mu$

The Rayleigh scattering is only of minor importance at $1.67 \mu$. The aerosol attenuation coefficient is larger than the Rayleigh below $8 \mathrm{~km}$ and above $15 \mathrm{~km}$ when the Elterman aerosol distribution is used. There is no ozone absorption. The optical thickness of the atmosphere is 0.123 . The reflected and transmitted radiance for $\mu_{0}=-1$ is shown in Fig. 10. Two different factors in these curves are characteristic of scattering primarily from an aerosol (Haze C) rather than from a Rayleigh scattering function ${ }^{10}$ : ( 1 ) the large variation in the reflected radiance as $A$ varies from 0 to $1 ;$ (2) the increase in the transmitted radiance near the zenith due to numerous small angle collisions.

The reflected and transmitted radiances for $\mu_{0}=-0.1$ are shown in Fig. Il. The following factors in these curves are characteristic of scattering primarily from an aerosol (Haze C) rather than from a Rayleigh scattering function ${ }^{10}$ : (1) for all albedos the reflected and transmitted radiances are considerably greater on the solar horizon than on the antisolar horizon; (2) the reflected radiance for $\mathrm{A}=0$ varies by several orders of magnitude from the solar horizon to the nadir; (3) the reflected radiance for $A=1$ is nearly constant from the antisolar horizon to the nadir; (4) the relatively small variation of the transmitted radiance with albedo particularly from the solar horizon to the zenith.

Mean Optical Path

The mean optical path of both the reflected and transmitted photons is given in Table $I$. The reflected mean optical path is lower at $0.27 \mu$ 
than at $0.30 \mu$ since the ozone absorption is much stronger at the former wavelength. As the wavelength increases the reflected mean optical path decreases as the optical thickness of the atmosphere decreases. The siight relative increase at $1.67 \mu$ is due to the predominance of aerosol scattering at this wavelength. For comparison three different values are listed at $0.70 \mu$ : (1) with ozone absorption and Elterman aerosol distribution; (2) no ozone absorption and Elterman distribution; (3) no ozone absorption and Kondratiev distribution. The transmitted mean optical path decreases uniformly with wavelength.

Flux

The flux at the lower boundary when $A=0$ (normalized to unit incident flux) is given in Table $I$. The tabulated flux includes the incident beam. The flux increases uniformly with wavelength for the values tabulated here. It is determined primarily by the strong ozone absorption in the ultraviolet and the rapidly decreasing importance of Rayleigh scattering as the wavelength increases. The variation with angle is interesting. For example, at $1.67 \mu$ the flux is 0.9837 when $\mu_{0}=-1$, but is only 0.6811 when $\mu_{0}=-0.1$.

Planetary Albedo

The planetary albedo is given in Table I both for a surface albedo $A=0$ and $A=0.8$. When $A=0$, the sum of the planetary albedo and the flux at the lower boundary is unity provided that there is no absorption. 
This may be verified at the wavelengths where there is no ozone absorption. When $\mu_{0}=-1$ and $A=0$, the maximum planetary albedo is 0.180 at $0.40 \mu$; when $\mu_{0}=-0.1$ and $A=0$, the maximum planetary albedo is 0.599 also at $0.40 \mu$. When $A=0.8$, the planetary albedos at a given $\mu_{0}$ show little variation between $0.4,0.7$, and $1.67 \mu$. They are still very small at the wavelengths with strong ozone absorption. The planetary albedo increases appreciably in the visible and infrared as the solar incident angle approaches the horizon. 
Table I.

Mean optical path, flux at lower boundary and planetary albedo.

\begin{tabular}{|c|c|c|c|c|c|c|c|}
\hline $\begin{array}{l}\text { Wavelength } \\
(\mu)\end{array}$ & $\begin{array}{l}\text { Optical } \\
\text { Thickness }\end{array}$ & $-\mu_{0}$ & $\begin{array}{l}\text { Reflected } \\
\text { mean optical } \\
\text { path }\end{array}$ & $\begin{array}{l}\text { Transmitted } \\
\text { mean optical } \\
\text { path }\end{array}$ & $\begin{array}{l}\text { Flux at } \\
\text { lower } \\
\text { boundary, } \\
A=0\end{array}$ & $\begin{array}{l}\text { Albedo } \\
\text { of planet, } \\
A=0\end{array}$ & $\begin{array}{l}\text { Albedo } \\
\text { of planet, } \\
A=0.8\end{array}$ \\
\hline 0.27 & 73.25 & 1 & 4.36 & --- & ----- & 0.00044 & 0.00044 \\
\hline 0.30 & 4.97 & 1 & 5.00 & 9.30 & 0.0136 & 0.00437 & 0.00443 \\
\hline 0.40 & 0.577 & 1 & 1.68 & 1.40 & 0.8204 & 0.180 & 0.790 \\
\hline 0.70 & $0.217^{1}$ & 1 & 0.938 & 0.454 & 0.9532 & 0.0382 & 0.775 \\
\hline 0.70 & $0.1433^{2}$ & 1 & 0.654 & 0.305 & 0.9711 & 0.0289 & 0.794 \\
\hline 0.70 & $0.1433^{3}$ & 1 & 0.648 & 0.317 & 0.9703 & 0.0297 & 0.794 \\
\hline 1.67 & 0.123 & 1 & 0.686 & 0.232 & 0.9837 & 0.0163 & 0.795 \\
\hline 0.27 & 73.25 & 0.1 & 2.56 & --- & ----- & 0.00128 & 0.00128 \\
\hline 0.30 & 4.97 & 0.1 & 3.30 & 10.61 & 0.000033 & 0.00986 & 0.00986 \\
\hline 0.40 & 0.577 & 0.1 & 2.23 & 2.69 & 0.4009 & 0.599 & 0.897 \\
\hline 0.70 & $0.1433^{2}$ & 0.1 & 1.17 & 1.18 & 0.6433 & 0.358 & 0.864 \\
\hline 1.67 & 0.123 & 0.1 & 1.10 & 1.09 & 0.6811 & 0.319 & 0.858 \\
\hline
\end{tabular}

Elterman aerosol distribution unless otherwise stated.

${ }^{1}$ Includes ozone absorption

${ }^{2}$ No ozone absorption

${ }^{3}$ No ozone absorption; Kondratiev aerosol distribution 
References

1. Coulson, K. L., J. V. Dave, and Z. Sekera, Tables Related to Radiation Emerging from a Planetary Atmosphere with Rayleigh Scattering (University of California Press, Los Angeles, 1960).

2. Feigelson, E. M., M. S. Malkevich, S. Ya. Kogan, T. D. Kononatova, K. S. Glazova, and M. A. Kunznetsova, Calculation of the Brightness of Light in the Case of Anisotropic Scattering (Consultants Bureau, Inc., New York, 1960).

3. Fraser, R. S., J. Opt. Soc. Amer. 54, 157 (1964).

4. Plass, G. N., and G. W. Kattawar, J. Geoph. Res. (in press).

5. Collins, D. G., K. Cunningham, and M. B. Wells, Monte Carlo Studies of Light Transport, Interim Report No. 2 (Radiation Research Asscciates, Inc., Font Worth, 1967).

6. Elterman, L., Handbook of Geophysics and Space Environments (S. L. Valley, Editor, Air Force Cambridge Research Laboratories, Bedford, Mass., 1965).

7. Kondratiev, K. Ya., G. A. Nicolsky, I. Ya. Badinov, and S. D. Andreev, Appl. Opt. 6 , 197 (1967).

8. Deirmendjian, D., Appl. Opt. $\underline{3}, 187$ (1964).

9. Kattawar, G. W., and G. N. Plass, Appl. Opt. $\underline{6}, 1377$ (1967); $\underline{6}, 1549(1967)$

10. Kattawar, G. W., and G. N. Plass, Appl. Opt. (in press).

11. Plass, G. N. and G. W. Kattawar, Appl. Opt. (in press). 


\section{Legends for Figures}

Fig. 1. Scattering function as a function of scattering angle for Haze $C$ model at a wavelength of $0.4,0.7$, and $1.67 \mu$. The inset in the upper left shows the function near $0^{\circ}$.

Fig. 2. Reflected and transmitted radiance at the top and bottom respectively of the earth's atmosphere for a wavelength of $0.7 \mu$ as a function of the cosine of the zenith angle $(\mu)$ for an incident solar angle whose cosine $\mu_{0}=-1$. Curves are given for various surface albedos $A$ and for the aerosol distributions of Elterman ${ }^{6}$ and Kondratiev et $a 1^{7}$. The optical depth $\tau$ of the atmosphere is 0.1433 . All radiance values in this paper are normalized to unit incident flux.

Fig. 3. Upward radiance for a wavelength of $0.7 \mu$ and $A=0$ at various heights in the atmosphere corresponding to $\tau=0$ (top of atmosphere), $0.005,0.01,0.02$, and 0.05 . Curves are given for the aerosol distributions of Elterman ${ }^{6}$ and Kondratiev et $\mathrm{al}^{7}$. The downward radiance on the right side of the figure is for $A=0.4$ and height corresponding to $\tau=0.005,0.01,0.02,0.05$, and 0.1433 (solid circles).

Fig. 4. The reflected and transmitted radiance at the top and bottom respectively of the earth's atmosphere for a wavelength of $0.7 \mu$ and $\mu_{0}=-0.1$. Curves are given for various surface albedos A. The Elterman aerosol distribution is used. The solar horizon is on the left of all graphs, the zenith or nadir is at the center, and the antisolar horizon at the right. The radiance for each $\mu$ interval has been averaged over all azimuth angles within $90^{\circ}$ of the incident plane. 
Fig. 5. Reflected and transmitted radiance for a wavelength of $0.4 \mu$ and $\mu_{0}=-1$. The optical depth $\tau=0.577$. The Elterman aerosol distribution is used. Curves are given for various surface albedos $\mathrm{A}$.

Fig. 6. Reflected and transmitted radiance for a wavelength of $0.4 \mu$ and $\mu_{0}=-0.1$ and the Elterman aerosol distribution. See caption to Fig. 4 .

Fig. 7. Reflected radiance for a wavelengths of $0.30 \mu$ and $0.27 \mu$ and $\mu_{0}$ -1 . The optical depth $\tau=4.97$ and 73.25 at the two wavelengths respectively. The curves are the same for all surface albedos. See caption to Fig. 5 .

Fig. 8. Transmitted radiance for a wavelength of $0.30 \mu$ and $\mu_{0}=-1$. See caption to Fig. 5 .

Fig. 9. Reflected radiance for wavelengths of $0.30 \mu$ and $0.27 \mu$ and $\mu_{0}=-0.1$. The curves are the same for all surface albedos. See caption to Fig. 4.

Fig. 10. Reflected and transmitted radiance for a wavelength of $1.67 \mu$ and $\mu_{0}=-1$. See caption to Fig. 5 .

Fig. 11. Reflected and transmitted radiance for a wavelength of $1.67 \mu$ and $\mu_{0}=-0.1$. See caption to Fig. 4. 


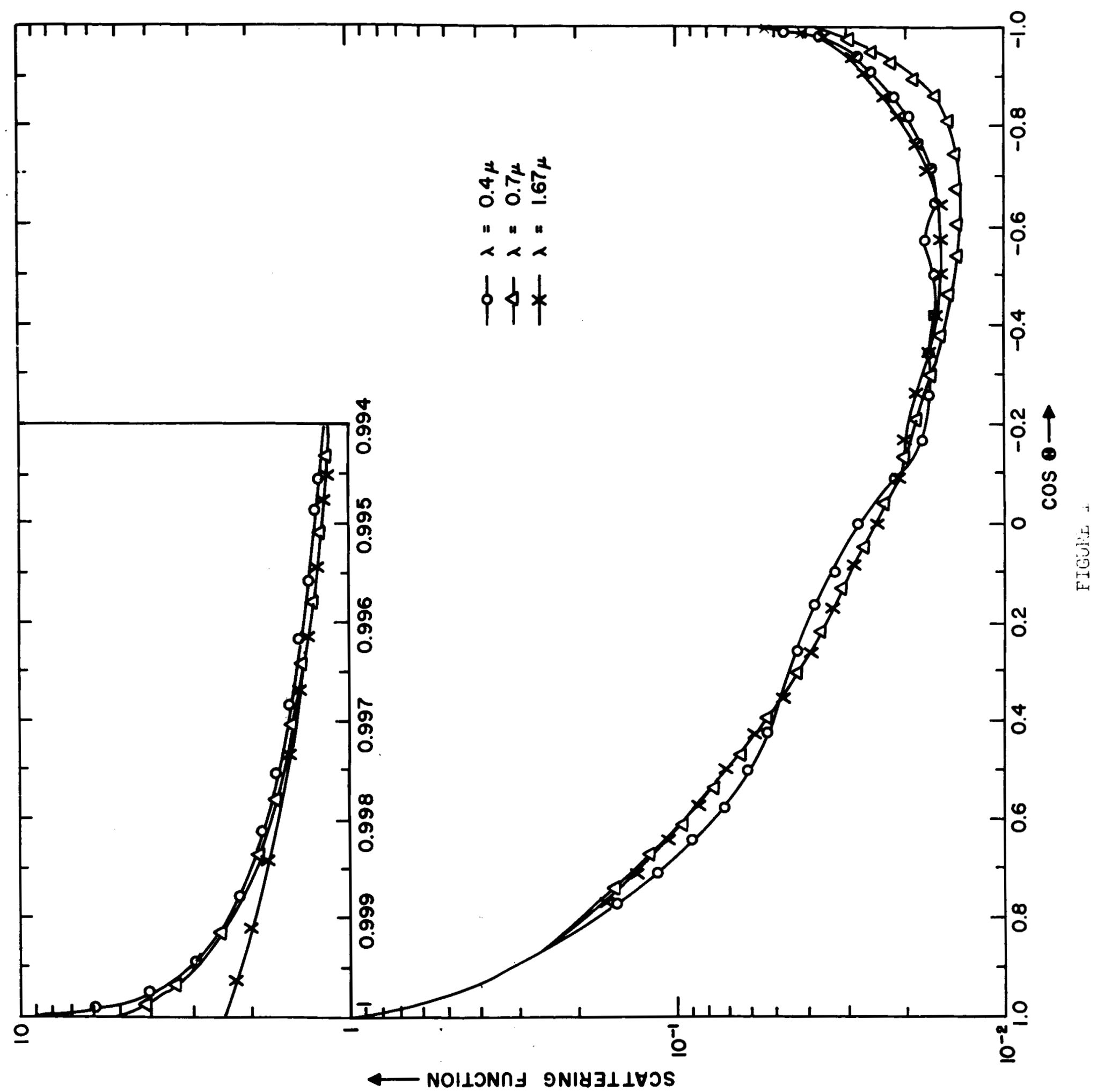



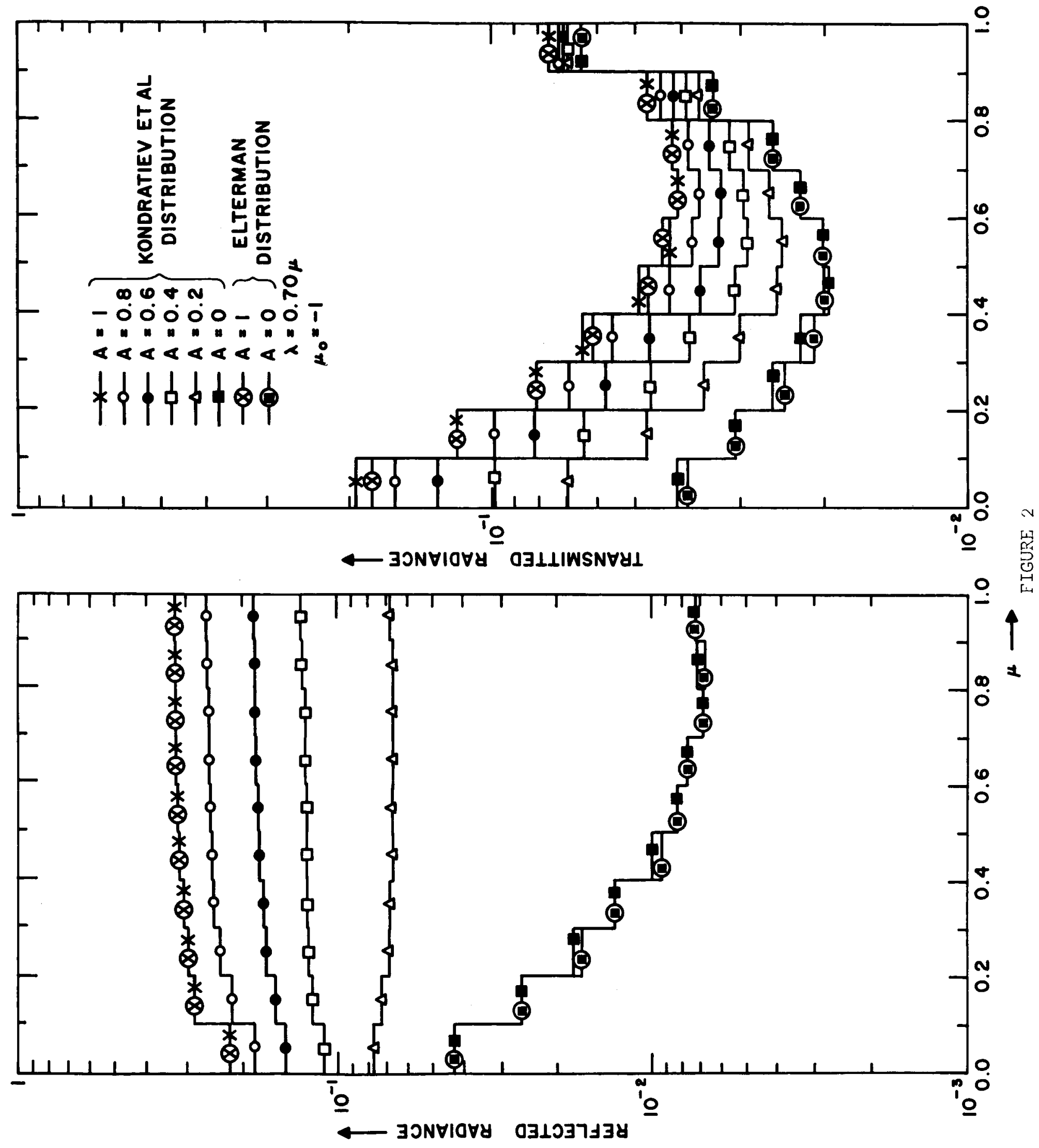

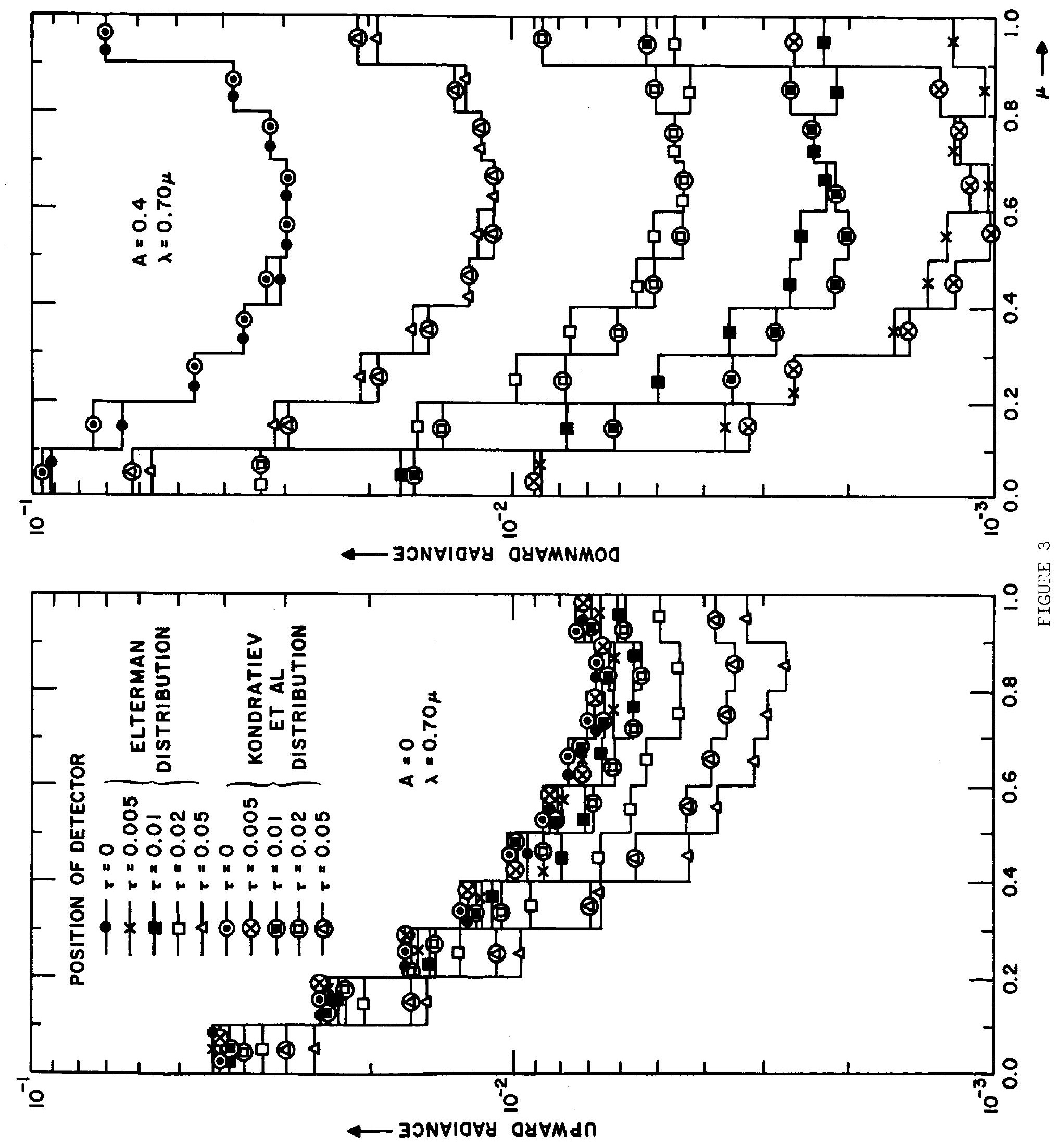


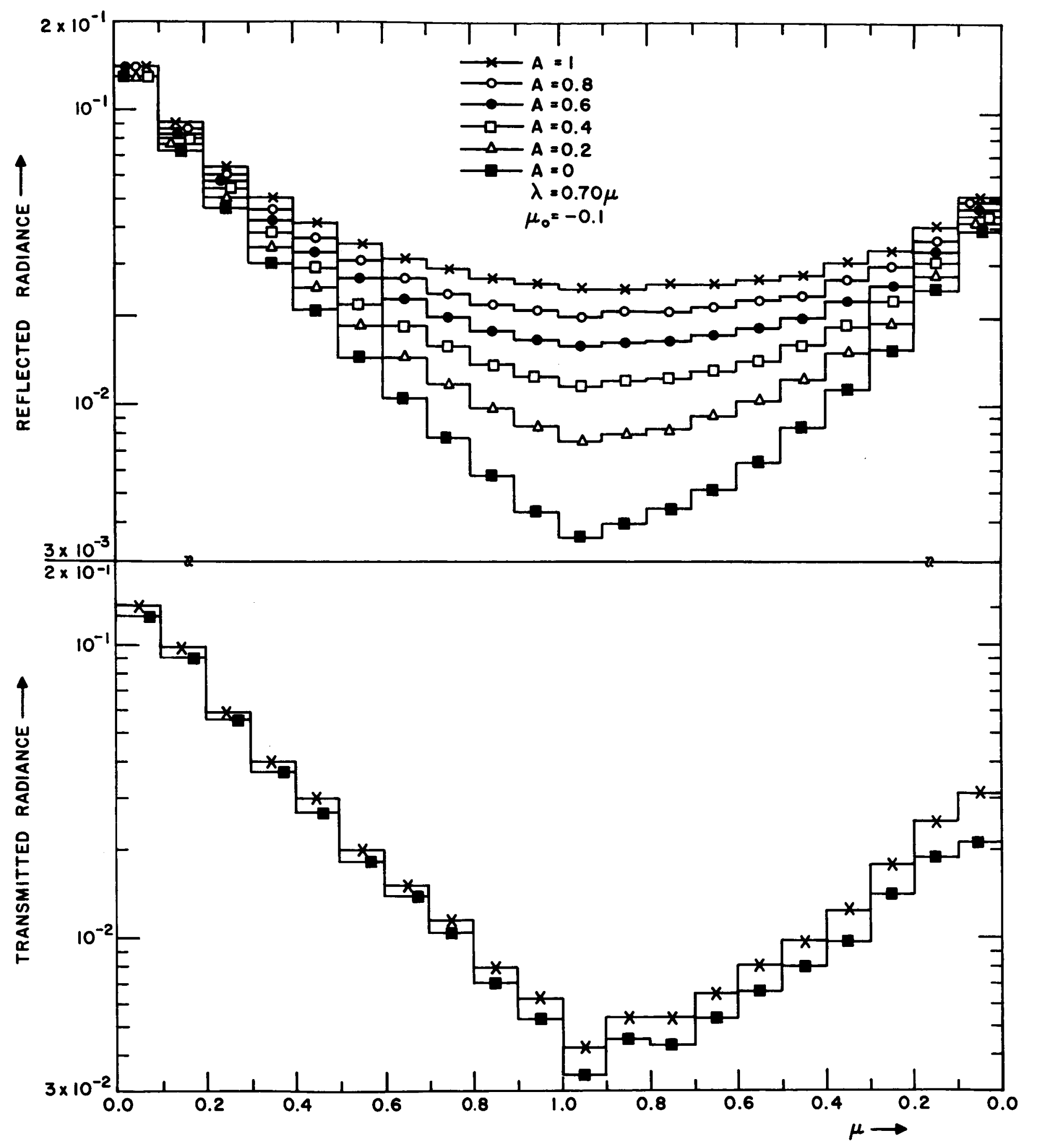



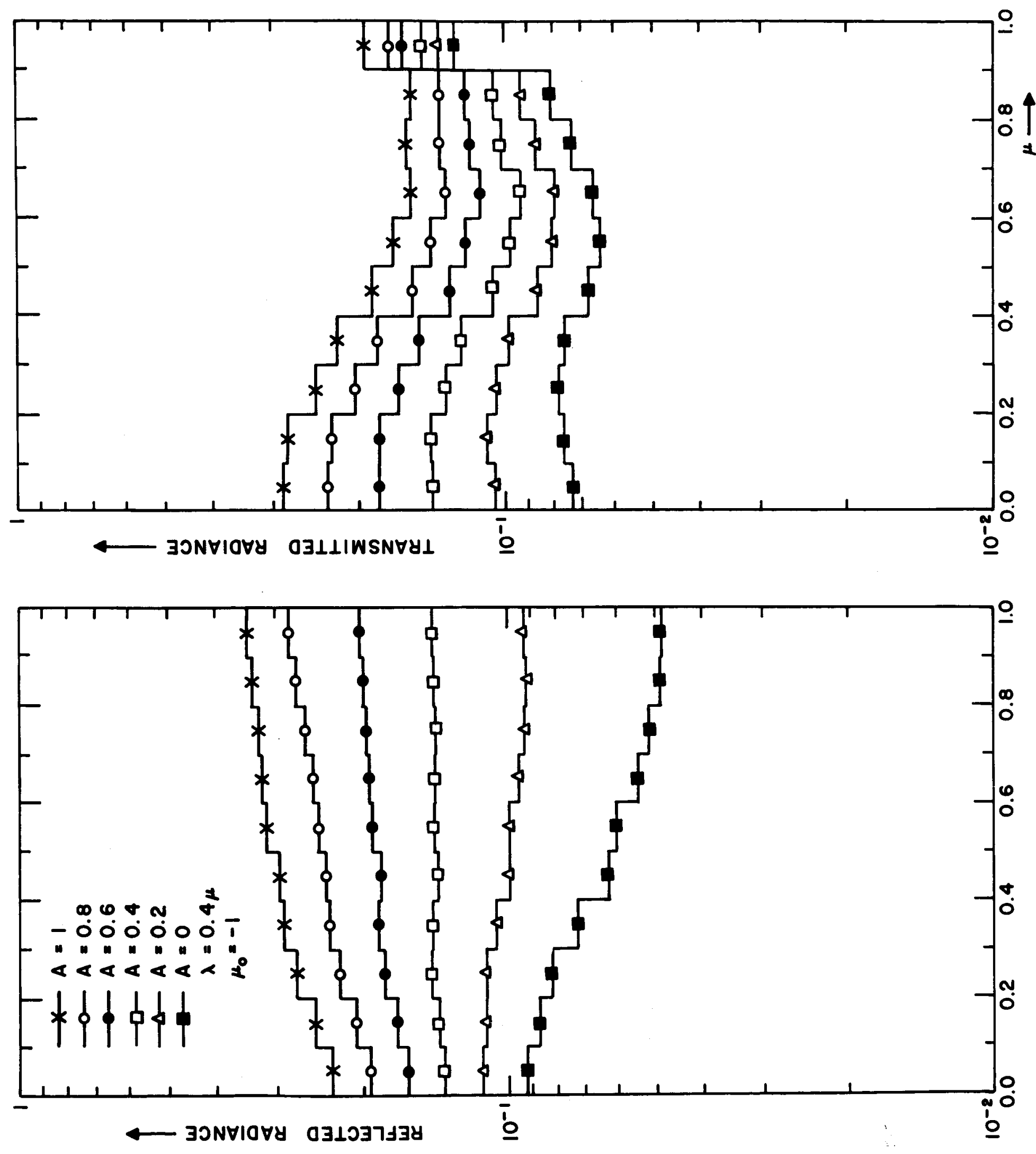


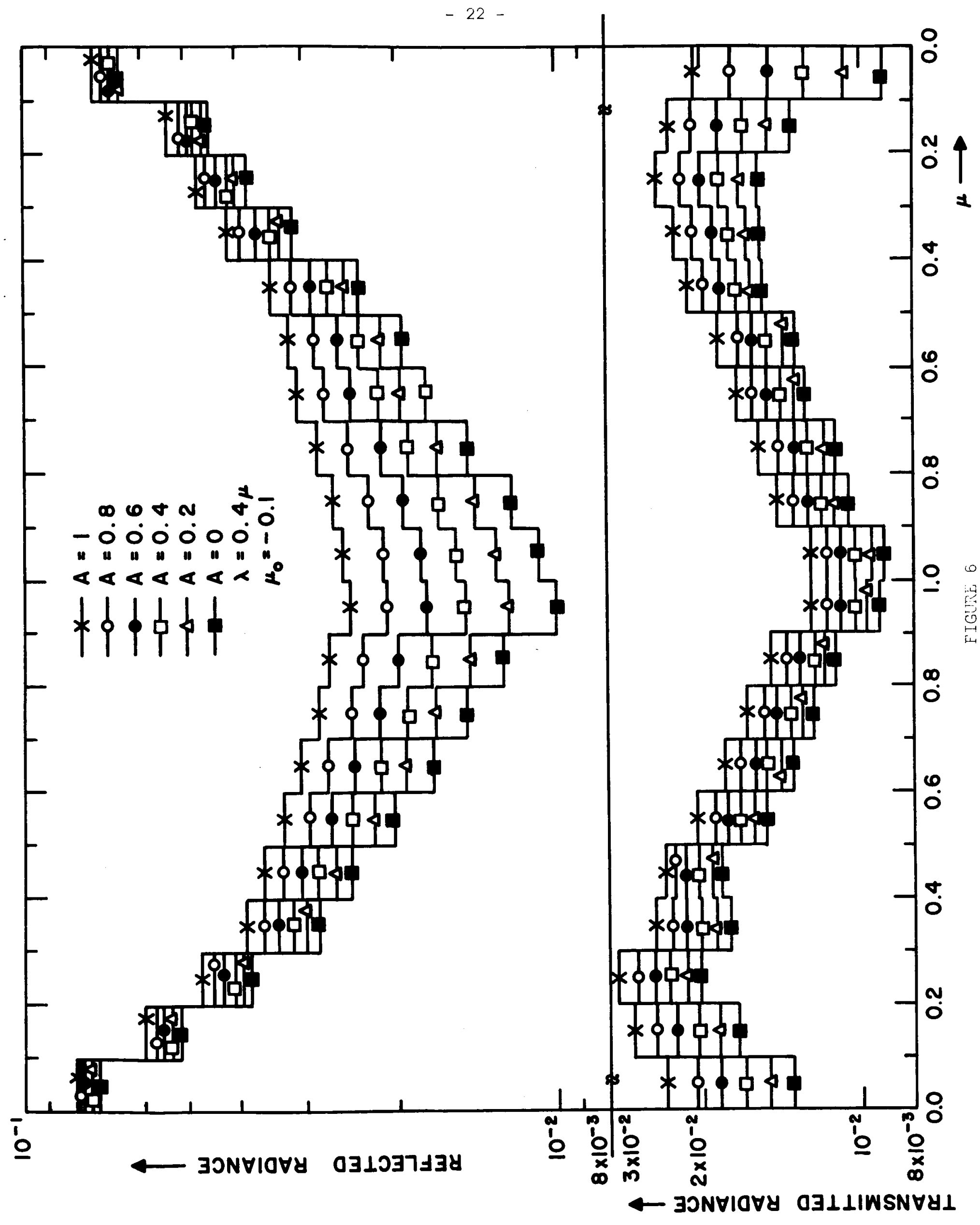




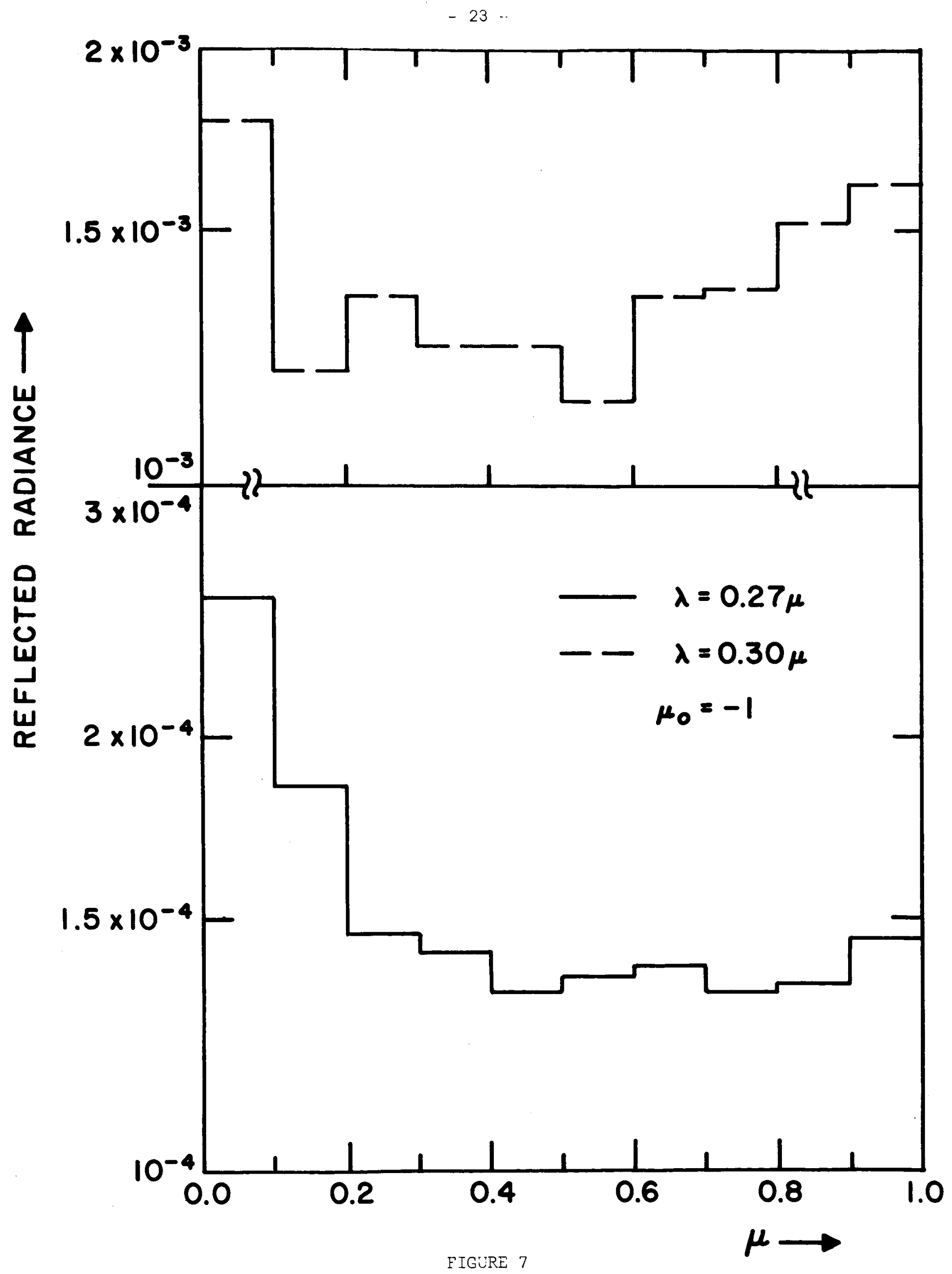




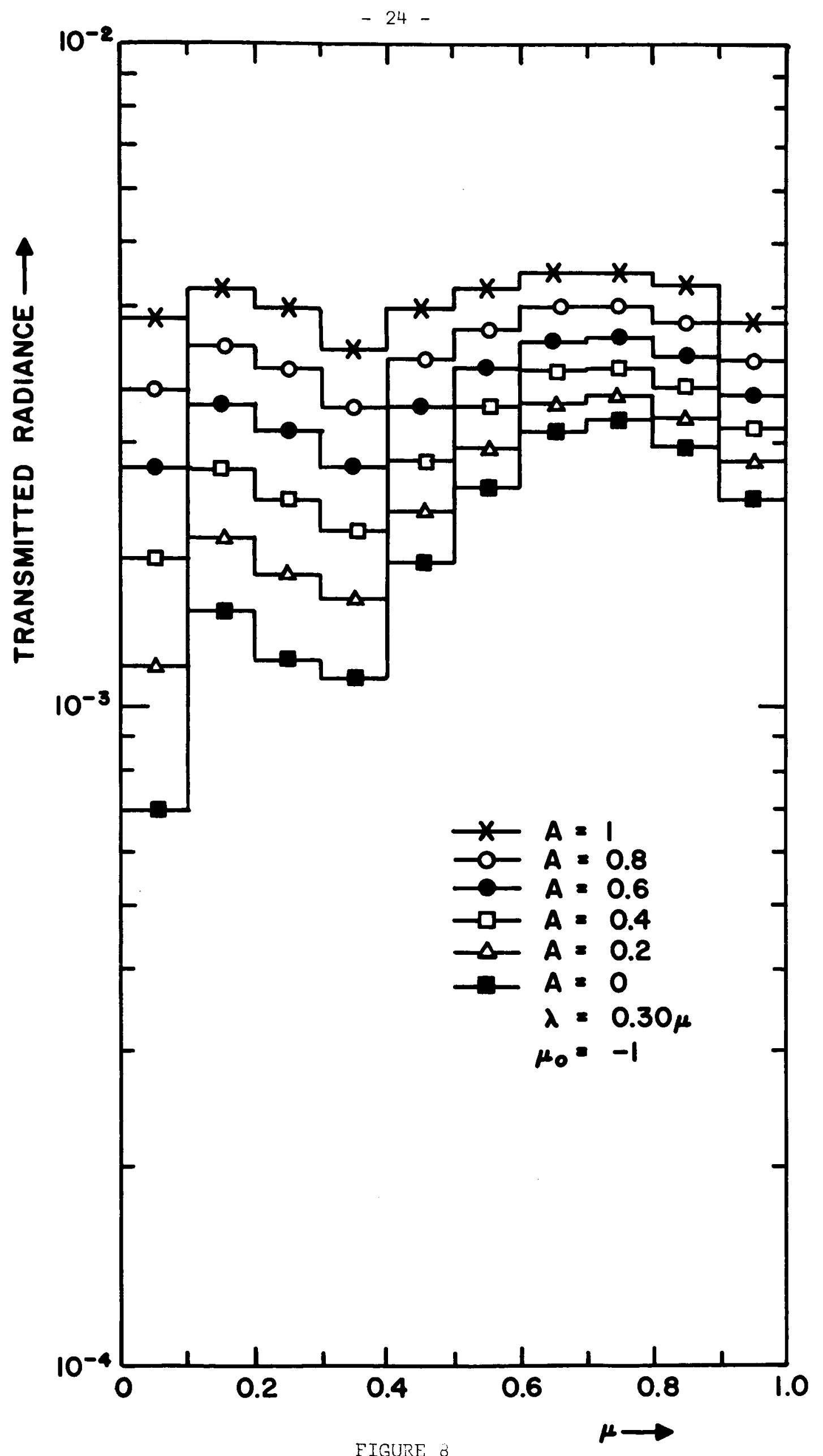




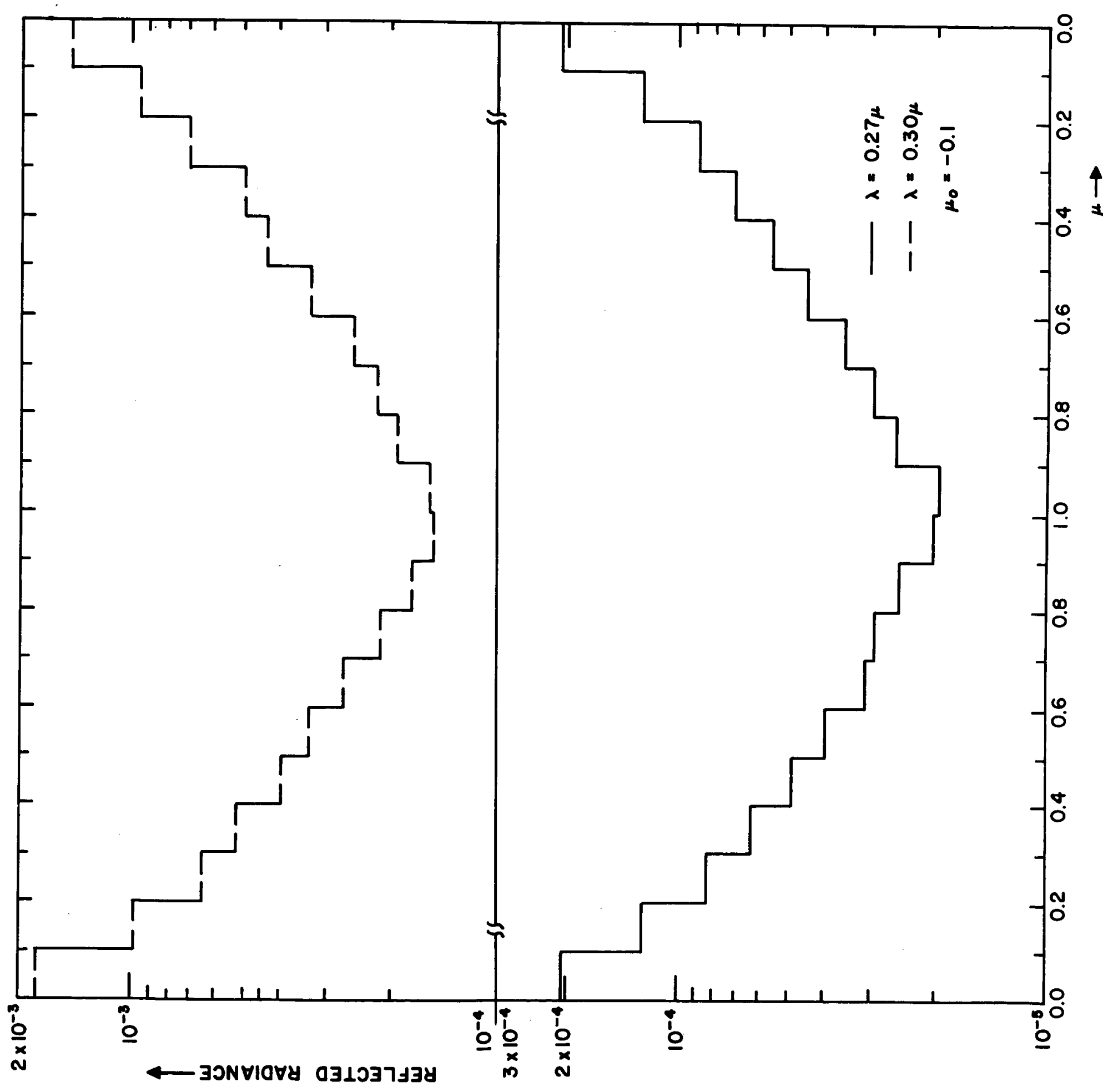

FIGURE 9 

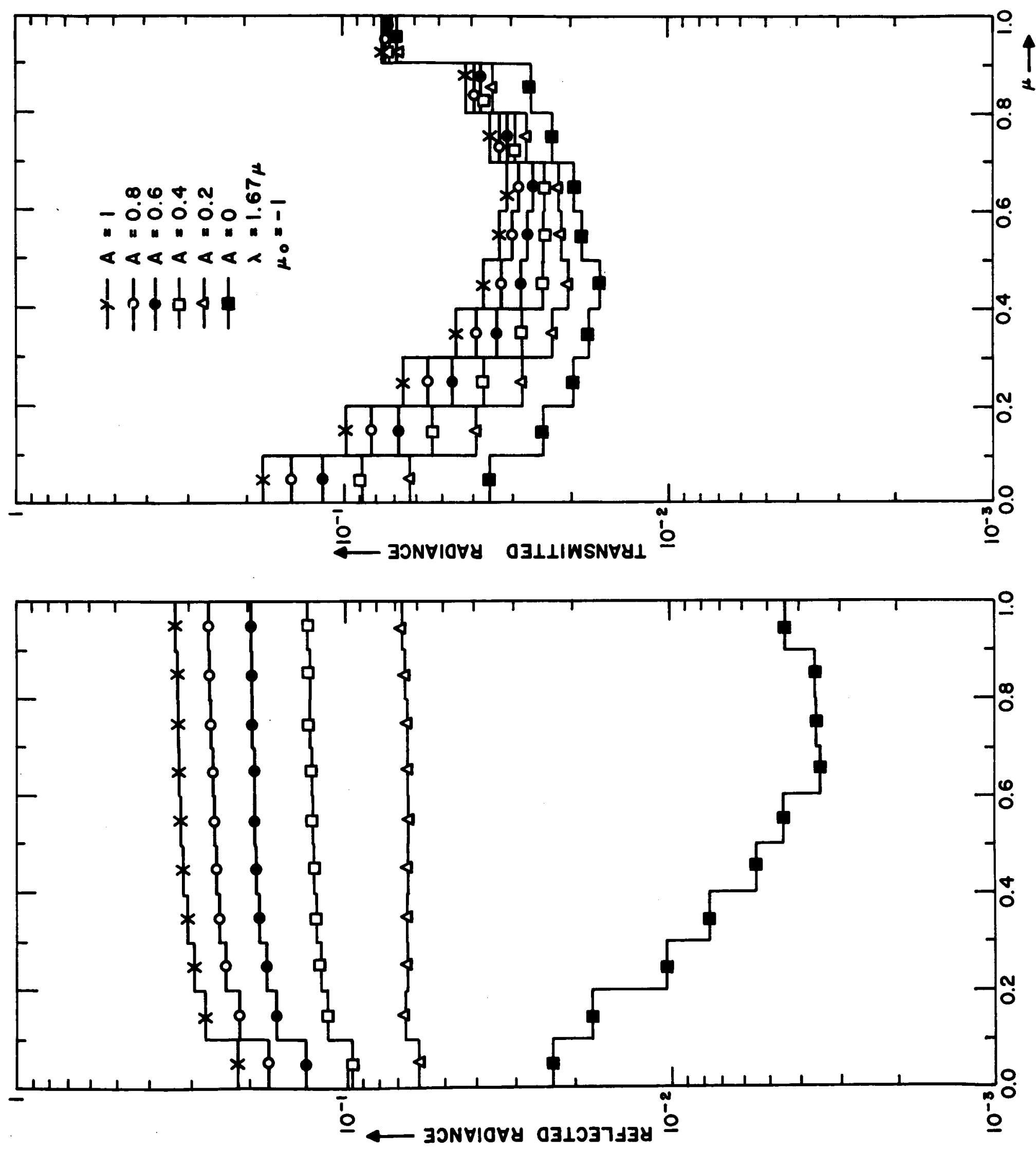


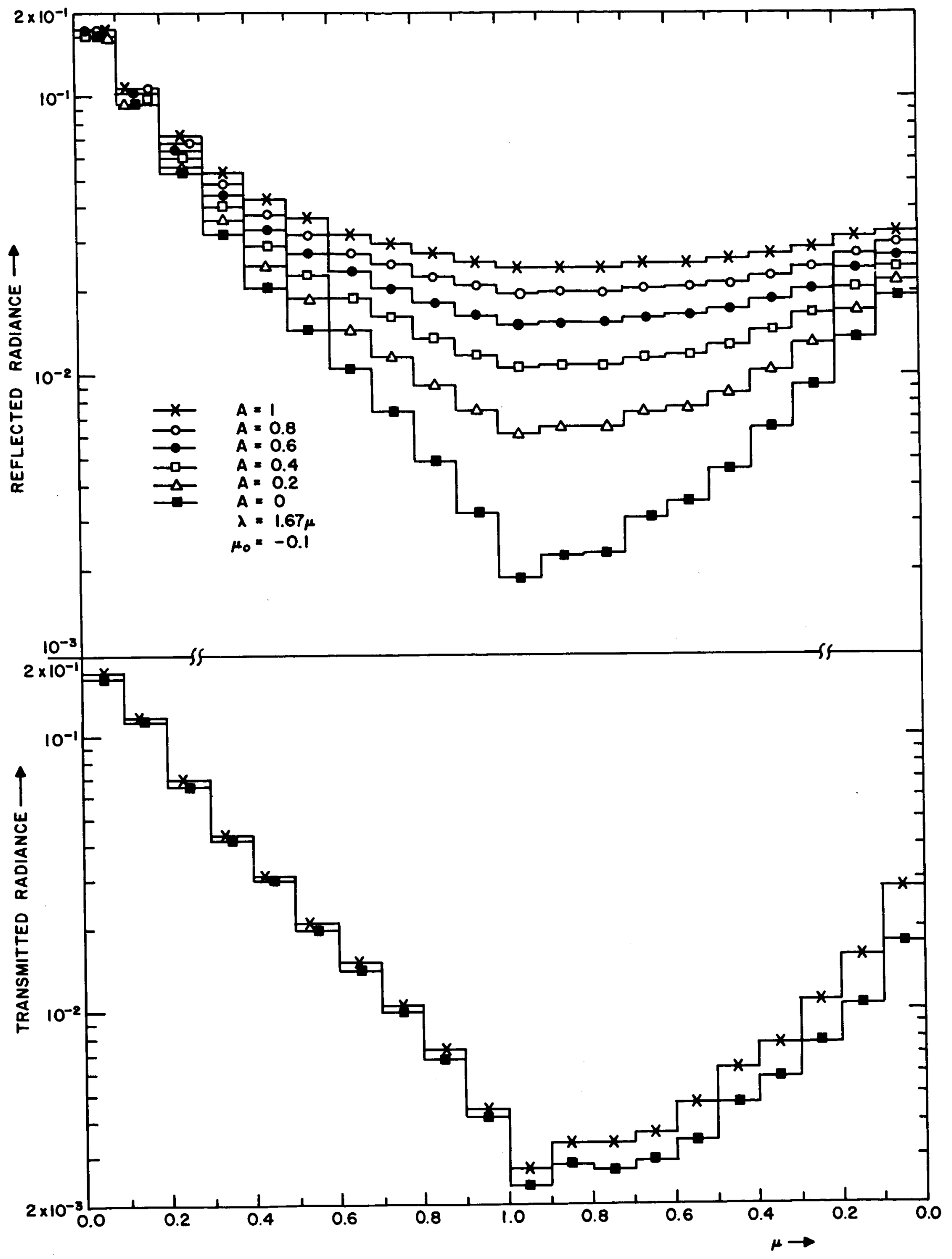

